\title{
Universal Natural Language Processing with Limited Annotations: Try Few-shot Textual Entailment as a Start
}

\author{
Wenpeng Yin ${ }^{1}$, Nazneen Fatema Rajani ${ }^{1}$, \\ Dragomir Radev ${ }^{1,2}$, Richard Socher ${ }^{1}$ and Caiming Xiong ${ }^{1}$ \\ ${ }^{1}$ Salesforce Research and ${ }^{2}$ Yale University \\ wyin@salesforce.com
}

\begin{abstract}
A standard way to address different NLP problems is by first constructing a problem-specific dataset, then building a model to fit this dataset. To build the ultimate artificial intelligence, we desire a single machine that can handle diverse new problems, for which task-specific annotations are limited. We bring up textual entailment as a unified solver for such NLP problems. However, current research of textual entailment has not spilled much ink on the following questions: (i) How well does a pretrained textual entailment system generalize across domains with only a handful of domainspecific examples? and (ii) When is it worth transforming an NLP task into textual entailment? We argue that the transforming is unnecessary if we can obtain rich annotations for this task. Textual entailment really matters particularly when the target NLP task has insufficient annotations.
\end{abstract}

Universal $\mathrm{NLP}^{1}$ can be probably achieved through different routines. In this work, we introduce Universal Few-shot textual Entailment (UFO-ENTAIL). We demonstrate that this framework enables a pretrained entailment model to work well on new entailment domains in a few-shot setting, and show its effectiveness as a unified solver for several downstream NLP tasks such as question answering and coreference resolution when the end-task annotations are limited. Code: https://github.com/ salesforce/UniversalFewShotNLP

\section{Introduction}

Nowadays, the whole NLP journey has been broken down into innumerable sub-tasks. We often solve

\footnotetext{
" "Universal NLP" here means using a single machine to address diverse NLP problems. This is different from using the same machine learning algorithm such as convolution nets to solve tasks because the latter still results in task-specific models which can not solve other tasks.
}

each task separately by first gathering task-specific training data and then tuning a machine learning system to learn the patterns in the data. Constrained by the current techniques, the journey has to be performed in this way. By a forward-looking perspective, instead, a single machine that can handle diverse (seen and unseen) tasks is desired. The reason is that we cannot always rely on expensive human resources to annotate large-scale task-specific labeled data, especially considering the inestimable number of tasks to be explored. Therefore, a reasonable attempt is to map diverse NLP tasks into a common learning problem-solving this common problem equals to solving any downstream NLP tasks, even some tasks that are new or have insufficient annotations.

Textual entailment (aka. natural language inference in Bowman et al. (2015)) is the task of studying the relation of two assertive sentences, Premise $(\mathrm{P})$ and Hypothesis $(\mathrm{H})$ : whether $\mathrm{H}$ is true given $\mathrm{P}$. Textual entailment (TE) was originally brought up as a unified framework for modeling diverse NLP tasks (Dagan et al., 2005; Poliak et al., 2018). The research on TE dates back more than two decades and has made significant progress. Particularly, with the advances of deep neural networks and the availability of large-scale human annotated datasets, fine-tuned systems often claim surpassing human performance on certain benchmarks.

Nevertheless, two open problems remain. First, the increasing performances on some benchmarks heavily rely on rich human annotations. There is rarely a trained entailment system that can work on benchmarks in other domains. Current textual entailment systems are far from being deployed in new domains where no rich annotation exists. Second, there is an increasing awareness in the community that lots of NLP tasks can be studied in the entailment framework. But it is unclear when it is worth transforming a target NLP tasks to textual 
entailment. We argue that textual entailment particularly matters when the target NLP task has insufficient annotations; in this way, some NLP tasks that share the same inference pattern and annotations are insufficient to build a task-specific model can be handled by a unified entailment system.

Motivated by the two issues, we build UFOENTAIL- the first ever generalized few-shot textual entailment system with the following setting. We first assume that we can access a largescale generic purpose TE dataset, such as MNLI (Williams et al., 2018); this dataset enables us to build a base entailment system with acceptable performance. To get even better performance in any new domain or new task, we combine the generic purpose TE dataset with a couple of domain/taskspecific examples to learn a better-performing entailment for that new domain/task. This is a reasonable assumption because in the real-world, any new domain or new task does not typically have large annotated data, but obtaining a couple of examples is usually feasible.

Technically, our UFO-ENTAIL is inspired by the Prototypical Network (Snell et al., 2017), a popular metric-based meta-learning paradigm, and the STILTS (Phang et al., 2018), a framework that makes use of pretraining on indirect tasks to help the target task. UFO-ENTAIL consists of a RoBERTa (Liu et al., 2019) encoder and a proposed cross-task nearest neighbor block. The RoBERTa, pretrained on MNLI, provides a representation space biased to the source domain; the cross-task nearest neighbor block is in charge of mitigating the distribution difference between the source domain and the target task (given only a few examples).

In experiments, we apply UFO-ENTAIL trained on MNLI and $k$ examples from the target domain/task to two out-of-domain entailment benchmarks and two NLP tasks (question answering and coreference resolution). Results show the effectiveness of UFO-ENTAIL in addressing the challenges set forth in the two questions. Overall, we make two contributions:

- We are the first to systematically study textual entailment in open domains, given only a couple of domain-specific examples.

- We follow the argument of some literature that textual entailment is a unified NLP framework. Here, we make a step further by declaring that we study textual entailment not because some NLP tasks can be transformed into entailment, but because few-shot textual entailment can be a promising attempt for universal NLP when we can not guarantee the accessibility of rich annotations.

\section{Related Work}

Textual Entailment. Textual entailment was first studied in Dagan et al. (2005) and the main focus in the early stages was to study lexical and some syntactic features. In the past few years, the research on textual entailment has been driven by the creation of large-scale datasets, such as SNLI (Bowman et al., 2015), science domain SciTail (Khot et al., 2018), and multi-genre MNLI (Williams et al., 2018). Representative work includes the first attentive recurrent neural network (Rocktäschel et al., 2016) and its followers (Wang and Jiang, 2016; Wang et al., 2017), as well as the attentive convolutional networks such as attentive pooling (dos Santos et al., 2016) and attentive convolution (Yin and Schütze, 2018), and self-attentive large-scale language models like BERT (Devlin et al., 2019) and RoBERTa (Liu et al., 2019). All these studies result in systems that are overly tailored to the datasets.

Our work differs in that we care more about fewshot applications of textual entailment, assuming that a new domain or an NLP task is not provided with rich annotated data.

Generalization via domain adaptation. Two main types of domain adaptation (DA) problems have been studied in literature: supervised DA and semi-supervised DA. In the supervised case, we have access to a large annotated data in the source domain and a small-scale annotated data in the target domain (Daumé III, 2007; Kang and Feng, 2018). In the semi-supervised case, we have a large but unannotated corpus in the target domain (Miller, 2019).

In contrast to semi-supervised DA, our work does not assume the availability of a large unlabeled data from the target domain or task. We also build more ambitious missions than the supervised DA since our work aims to adapt the model to new domains as well as new NLP tasks.

Generalization via few-shot learning. Fewshot problems are studied typically in the image domain (Koch et al., 2015; Vinyals et al., 2016; Snell et al., 2017; Ren et al., 2018; Sung et al., 2018). The core idea in metric-based few-shot 
learning is similar to nearest neighbors. The predicted probability of a test instance over a set of classes (i.e., only a few supporting examples are seen) is a weighted sum of classes for those supporting samples. Vinyals et al. (2016) compare each test instance with those supporting examples by the cosine distance in a method named Matching Networks. Snell et al. (2017) propose Prototypical Networks which first build prototypical representations for each class by summing up representations of supporting examples, then compare classes with test instances by squared Euclidean distances. Unlike fixed metric measures, the Relation Network (Sung et al., 2018) implements the comparison through learning a matching metric in a multi-layer architecture.

In the language domain, Yu et al. (2018) combine multiple metrics learned from diverse clusters of training tasks for an unseen few-shot text classification task. Han et al. (2018) release a few-shot relation classification dataset "FewRel" and compare a couple of representative methods on it.

These few-shot studies assume that, in the same domain, a part of the classes have limited samples, while other classes have adequate examples. In this work, we make a more challenging assumption that all classes in the target domain have only a couple of examples, and the training classes and testing classes are from different domains.

Unified natural language processing. McCann et al. (2018) cast a group of NLP tasks as question answering over context, such as machine translation, summarization, natural language inference, sequence modeling, etc. Raffel et al. (2019) study transfer learning for broad NLP by converting every language problem into a text-to-text format. Keskar et al. (2019) unify question answering, text classification, and regression via span extraction to get rid of various output layers on top of BERT for different tasks. A concurrent work with ours (Bansal et al., 2019) studies few-shot learning in NLP, but only text classification tasks are involved.

Their unification is mainly from the perspective of system structure, i.e., some distinct NLP tasks can be converted into a common training structure. Rich annotations are still needed. Our entailment paradigm, instead, is driven by the fact that there is a common reasoning pattern behind (Dagan et al., 2005). In addition, we care more about the challenges in realistic scenarios where we have to handle problems with limited annotations.
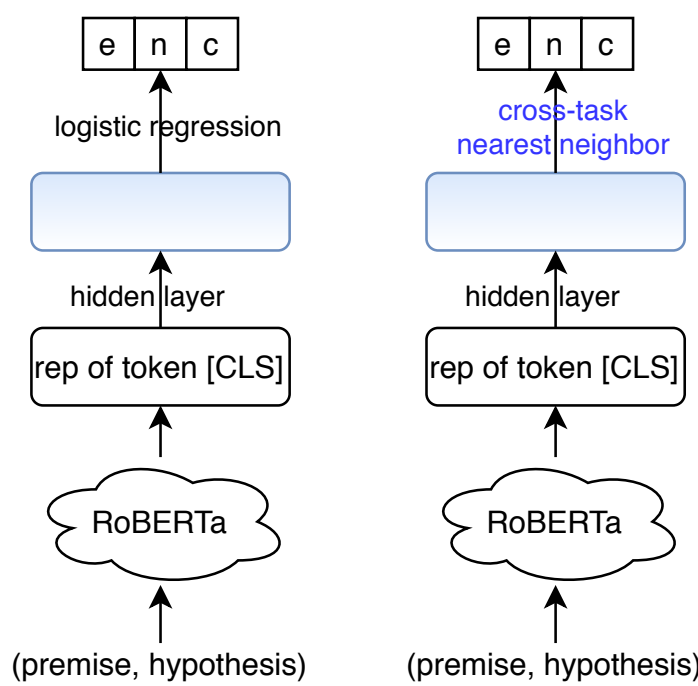

Figure 1: (Left) RoBERTa for textual entailment. "e": entailment, "n": neutral, "c": contradiction. (Right) the skeleton of our UFO-ENTAIL system. It basically replaces the logistic regression layer in RoBERTa classifier by a cross-task nearest neighbor block. RoBERTa learns class representations implicitly in the weight matrix of logistic regression, while UFO-ENTAIL first explicitly builds class representations for both source and target tasks, then composes the cross-task probability distributions to get the prediction.

\section{Method}

\subsection{Problem formulation}

Provided the large-scale generic textual entailment dataset MNLI (Williams et al., 2018) and a few examples from a target domain or a target task, we build an entailment predictor that can work well in the target domain/task even if only a few examples are available.

The inputs include: MNLI, the example set (i.e., $k$ examples for each type in \{ "entailment", "nonentailment"\} or \{ "entailment", "neutral", "contradiction" $\}$ if applicable). The output is an entailment classifier, predicting a label for each instance in the new domain/task. Please note that we need to convert those examples into labeled entailment instances if the target task is not a standard entailment problem. The entailment-style outputs can be easily converted to the prediction format required by the target tasks, as introduced in Section 4.2.

\subsection{Our model UFO-ENTAIL}

Hereafter, we refer to MNLI as $S$ (source domain), and the new domain or task as $T$. Before launching the introduction of UFO-ENTAIL, we first give a brief description: UFO-ENTAIL, shown in Figure 


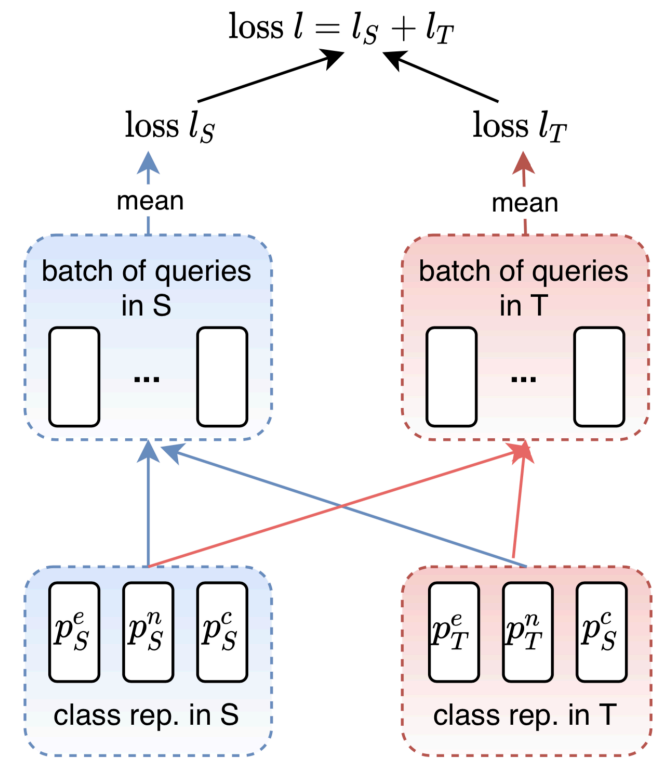

Figure 2: Loss computation in cross-domain/task nearest neighbor framework.

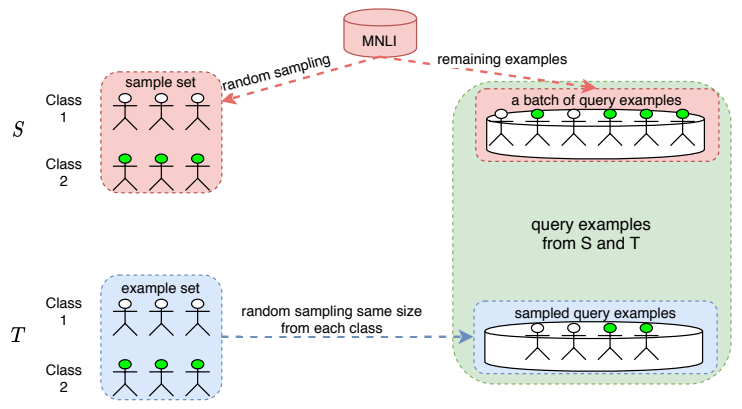

Figure 3: Generating the example set in $T$, the sample set in $S$ and the query examples by both $S$ and $T$. Here we only show two classes in $S$ and $T$ just for simplicity.

\section{1, is stacking a cross-task nearest neighbor block over a RoBERTa encoder.}

Encoder RoBERTa. For textual entailment, RoBERTa, shown in Figure 1, takes the pair (premise, hypothesis) as an input. RoBERTa first outputs a representation vector (e.g., the one corresponding to the token "CLS") to denote the input pair, then maps this representation into a new space by a hidden layer, finally conducts classification on that space through logistic regression. Overall, RoBERTa works with the hidden layer together as the encoder. For convenience, we still name this "RoBERTa+HiddenLayer" encoder as "RoBERTa".

We prepare RoBERTa by pretraining it on the source data $S$. This pretrained entailment encoder will act as a base system to deal with any new tasks (with the help of $k$ examples).
Cross-task nearest neighbor. Shown in Figure 2 , the first step in the cross-task nearest neighbor is to build representations for each class in the $S$ and $T$, then batches of query instances from $S$ as well as $T$ compare with those class representations by a matching function to compute loss and train the system. The reason we emphasize "cross-task" here is that both the classes and the query examples cover the two tasks $S$ and $T$. This is the core of UFO-ENTAIL in dealing with any new NLP problems of scarce annotations from textual entailment.

- Class representations. We use $p_{w}^{e}, p_{w}^{n}$ and $p_{w}^{c}$ to denote the representations for the three classes \{ "entailment", "neutral", "contradict"\} in $w, w \in\{S, T\}$. When the target task $T$ can only be converted into two classes, i.e., "entail vs. nonentail", we let $p_{T}^{n}=p_{T}^{c}$, both denoting the class "non-entail".

For the target $T$, each class has $k$ labeled examples (example set). For the source domain $S$, similar with the episode training in meta learning (Snell et al., 2017), we randomly sample $k$ examples ("sample set") of each class in $S$. Then,

$$
p_{w}^{j}=\frac{1}{k} \sum_{i=1}^{k} \operatorname{RoBERTa}\left(x_{n}^{i}\right)
$$

where $\left\{x_{n}^{i}\right\}, i=1 \cdots k$, are the labeled $k$ examples for class $j \in\{\mathrm{e}, \mathrm{n}, \mathrm{c}\}$ in $T$ or $S, \operatorname{RoBERTa}(\cdot) \in$ $\mathbb{R}^{d}$ and $p_{w}^{j} \in \mathbb{R}^{d}$. Overall, UFO-ENTAIL keeps representations for six classes.

- Query examples. As Figure 3 illustrates, a query batch is composed of two sub-batches, one from $S$, the other from $T$. For $S$, apart from its "sample set", the remaining labeled examples are grouped as mini-batches. For $T$, since all the labeled examples it has are those $k$ supporting examples per class, we randomly sample $m$ examples from the $k$ supporting examples for each class $(m<k)$, and finally incorporate them into a $S$ 's mini-batch as a bigger batch of queries.

Since UFO-ENTAIL is to cope with new tasks given a textual entailment task. We assume that the source entailment task provides valuable knowledge to warm up the model learning. For a testing instance in $T$, we want it to compose the reasoning conclusions derived from both $S$ and the example set in $T$.

For training, we include examples from $S$ as queries because we treat the classes in $S$ and $T$ equally, and the queries in $S$ and $T$ equally as well. This leads to a higher-level abstract task in which 


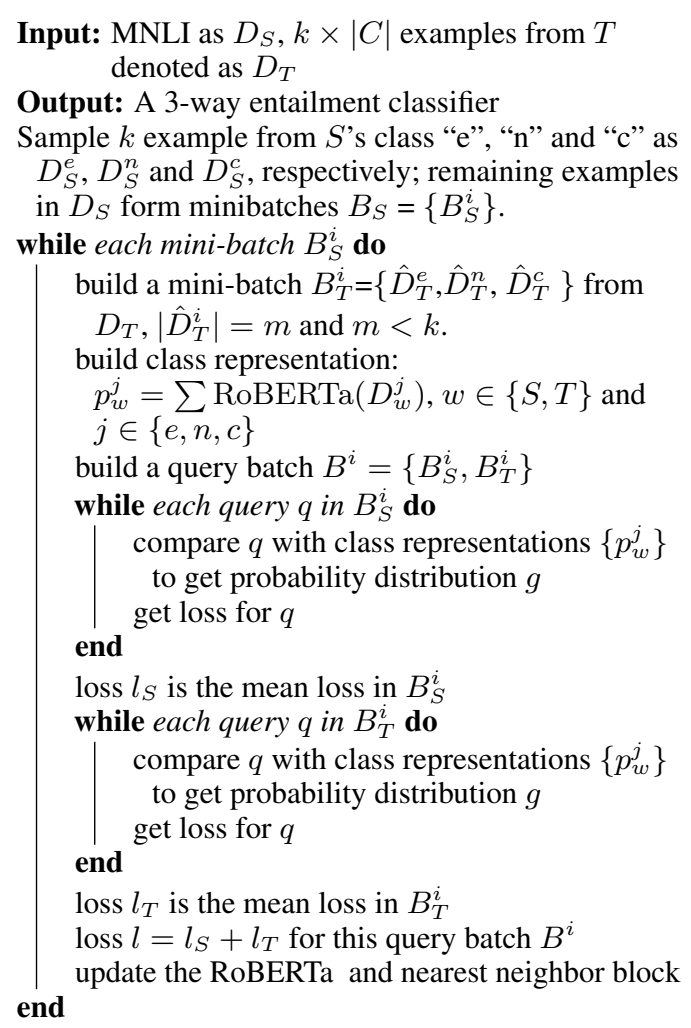

Algorithm 1: UFO-ENTAIL algorithm.

$S$ and $T$ learns from each other to mitigate the difference.

Matching function. Assuming a query example gets its representation $q$ through RoBERTa, then a matching score, between this query example and one class (class representation $p$ ), $s_{p, q}$ is learnt as follows:

$$
\begin{aligned}
I & =[p, q, p \circ q, p-q] \\
r_{1} & =\operatorname{dropout}\left(\tanh \left(W_{1} \times I\right)\right)+I \\
r_{2} & =\operatorname{dropout}\left(\tanh \left(W_{2} \times r_{1}\right)\right)+r_{1} \\
r_{3} & =\operatorname{dropout}\left(\tanh \left(W_{3} \times r_{2}\right)\right) \\
r_{4} & =\operatorname{dropout}\left(\tanh \left(W_{4} \times r_{3}\right)\right) \\
s_{p, q} & =\operatorname{sigmoid}\left(W_{5} \times r_{4}\right)
\end{aligned}
$$

where $I \in \mathbb{R}^{4 d}, W_{1}$ and $W_{2} \in \mathbb{R}^{4 d \times 4 d}, W_{3} \in$ $\mathbb{R}^{4 d \times 2 d}, W_{4} \in \mathbb{R}^{2 d \times d}$ and $W_{5} \in \mathbb{R}^{d}$.

Probability distribution per query. A query example will obtain three matching scores from $S$ $\left(g_{S} \in \mathbb{R}^{3}\right)$ and three matching scores from $T$ $\left(g_{T} \in \mathbb{R}^{3}\right)$. Now we try to combine them as a final probability distribution of thee dimensions. Instead of linear combination with artificial weights, we let the system learn automatically the contribution of $g_{S}$ and $g_{T}$ in a new space. Therefore, the final probability distribution $g \in \mathbb{R}^{3}$ is learned as follows:

$$
\begin{aligned}
\hat{g}_{S} & =\operatorname{sigmoid}\left(W_{6} \times g_{S}\right) \\
\hat{g}_{T} & =\operatorname{sigmoid}\left(W_{6} \times g_{T}\right) \\
\lambda & =\operatorname{sigmoid}\left(W_{7} \times\left[g_{S}, g_{T}\right]\right) \\
g & =\operatorname{softmax}\left(\lambda \circ \hat{g}_{S}+(1-\lambda) \circ \hat{g}_{T}\right)
\end{aligned}
$$

where $W_{6} \in \mathbb{R}^{3}$ and $W_{7} \in \mathbb{R}^{6} . g$ is used to compute loss to train the system in training and predict the class in testing.

Training loss. In training, a query batch actually contains two sub-batches, one from $S$, the other from $T$. To balance the contribution, we first compute the mean loss in $S$ 's and $T$ 's sub-batches respectively, obtaining $l_{S}$ and $l_{T}$, then the overall loss for that batch is $l=l_{S}+l_{T}$, demonstrated in Figure 2,

The whole UFO-ENTAIL system is a stack of the RoBERTa and the cross-task nearest neighbor block. Its learning algorithm is summarized in the Algorithm 1;. UFO-ENTAIL can be trained endto-end.

\subsection{UFO-ENTAIL vs. other related models}

- UFO-EnTail vs. Prototype. Net. Prototypical network (Snell et al., 2017) assumes that training tasks and test tasks are in the same distribution. So, it focuses on the matching function learning and hopes a well-trained matching function in training tasks (i.e., $S$ in this work) works well in the target tasks (i.e., $T$ here). However, the presumption does not apply to the cross-domain/task scenarios in this work.

Similarly, UFO-ENTAIL also builds class representation by averaging the representations of some class-specific labeled examples, as prototypical network does. In training, prototypical network builds class representations in training tasks and query examples come from the training tasks only; in testing, the query examples from the testing tasks only compare with the few labeled examples specific to the testing task (training tasks do not participate anymore). In short, prototypical network only builds nearest neighbor algorithm within a task. UFO-ENTAIL differs in that it is based on cross-task nearest neighbor - keeping class representations for both $S$ and $T$ in training as well as in testing; query examples in training also comes from $S$ and $T$. Because of the mismatch of the distributions in $S$ and $T$, the goal of UFO-ENTAIL is to not only learn the matching function, but also map the instances in $S$ and $T$ to the same space. 
UFO-EnTaIL vs. STILTS. Given the source data $S$ and a couple of labeled examples from the target $T$, STILTS (Phang et al., 2018) first trains RoBERTa on $S$, then fine-tune on the labeled examples of $T$. Both the pretraining and fine-tuning use the same RoBERTa system in Figure 1. It has been widely used as the state of the art technique for making use of related tasks to improve target tasks, especially when the target tasks have limited annotations (Liu et al., 2019; Sap et al., 2019; Clark et al., 2019). By the architecture, STILTS relies on the standard RoBERTa classifier which consists of a RoBERTa encoder and a logistic regression on the top; UFO-ENTAIL instead has a cross-task nearest neighbor block on the top of the RoBERTa encoder.

STILTS tries to learn the target-specific parameters by tuning on the $k$ labeled examples. However, this is very challenging if $k$ is over small, like values $\{1,3,5,10\}$ we will use in our problems. We can also think STILTS learns class prototypical representations implicitly (i.e., the weights in the logistic regression layer), however, the bias term in the logistic regression layer reflect mainly the distribution in the source $S$, which is less optimal for predicting in the target $T$.

\section{Experiments}

We apply UFO-ENTAIL to entailment tasks of open domain and open NLP tasks.

Experimental setup. Our system is implemented with Pytorch on the transformers package released by Huggingface ${ }^{2}$. We use "RoBERTalarge" initialized by the pretrained language model.

To mitigate the potential bias or artifacts ( $\mathrm{Gu}$ rurangan et al., 2018) in sampling, all numbers of $k$-shot are average of five runs in seeds $\{42,16,32$, $64,128\}$.

Due to GPU memory constraints, we only update the nearest neighbor block, the hidden layer and top-5 layers in RoBERTa. For other training configurations, please refer to our released code.

Baselines. The following baselines are shared by experiments on open entailment tasks and open NLP tasks.

- 0-shot. We assume zero examples from target domains. We train a RoBERTa classifier ${ }^{3}$ on

\footnotetext{
${ }^{2}$ https://github.com/huggingface/ transformers

${ }^{3}$ Specifically, the "RobertaForSequenceClassification" classifier in the Huggingface transformer.
}

MNLI, and apply it to the respective test set of target domains without fine-tuning.

- Train on $k$ examples. We build a RoBERTa classifier on the k labeled examples directly. No MNLI data is used. When $k$ is increased to cover all the labeled examples of the target domain or task, this baseline is referred as "train on target data".

- STILTs (Phang et al., 2018). This is a learning paradigm: for any target task, first pretrain the model on intermediate tasks, then fine-tune on the target task. Here, it means pretraining on MNLI, then fine-tuning on $k$ examples $(k>=1$ until it reaches the full labeled data of the target domain/task). When $k=0$, "STILTS" equals to "0-shot" baseline.

- Prototypical Network (Snell et al., 2017). It is a representative episode-training algorithms for few-shot problems, introduced in Section 2.

- State-of-the-art. STILTS is widely used as the state-of-the-art technique to promote the performance of a target problem with indirect supervision and task-specific fine-tuning. According to the definition of STILTS, its paradigm is applicable to any Transformer-based models. Since RoBERTa is used as the main Transformer model, applying STILTS to RoBERTa, which pretrains on MNLI then fine-tunes on the full target data, is the state of the art for this work.

\subsection{UFO-ENTAIL in open domains}

We test the few-shot setting on two out-of-domain entailment datasets: GLUE RTE (Wang et al., 2019) and SciTail (Khot et al., 2018). Examples in GLUE-RTE mainly come from the news and Wikipedia domains. SciTail is from the science domain, designed from the end task of multiple-choice QA. Our source dataset MNLI covers a broad range of genres such as conversation, news reports, travel guides, fundraising letters, cultural articles, fiction, etc. RTE has 2,490/277/2,999 examples in train/dev/test; SciTail has $23,596 / 1,304 / 2,126$ respectively.

\subsection{UFO-ENTAIL in open NLP tasks}

In this section, we apply UFO-ENTAIL as a universal framework to other distinct NLP tasks with limited annotations. An alternative approach to handle a task in which the annotations are scarce is to do transfer learning based on existing datasets of rich annotations and high relevance. However, we argue that this still results in "training separate models for different tasks", and it is unrealistic to 


\begin{tabular}{|c|c|c|c|c|c|}
\hline & & \multicolumn{2}{|c|}{ open entailment tasks } & \multicolumn{2}{|c|}{ open NLP tasks } \\
\hline \multicolumn{2}{|c|}{ \#entail-style pairs } & $\begin{array}{c}\text { RTE } \\
(2.5 \mathrm{k})\end{array}$ & $\begin{array}{c}\text { SciTail } \\
(23 \mathrm{k})\end{array}$ & $\begin{array}{c}\text { QA } \\
(4.8 \mathrm{k})\end{array}$ & $\begin{array}{c}\text { Coref. } \\
(4 \mathrm{k})\end{array}$ \\
\hline & "majority or random & 50.16 & 60.40 & 25.00 & 50.00 \\
\hline 0 -shot & train on MNLI & 83.36 & 81.70 & 58.00 & 61.76 \\
\hline \multirow{4}{*}{ 1-shot } & train on k examp. & $50.02 \pm 0.27$ & $48.14 \pm 8.00$ & $25.31 \pm 2.56$ & $51.14 \pm 0.42$ \\
\hline & prototype ne & $79.17 \pm 3.75$ & $75.13 \pm 7.60$ & $68.67 \pm 2.69$ & $61.91 \pm 17.5$ \\
\hline & STILTs & $83.86 \pm 0.25$ & $81.64 \pm 0.13$ & $63.20 \pm 3.55$ & $64.31 \pm 1.71$ \\
\hline & UFO-ENTAIL & $84.76 \pm 0.35$ & $83.73 \pm 1.10$ & $71.70 \pm 2.55$ & $74.20 \pm 3.14$ \\
\hline \multirow{4}{*}{ 3-shot } & train on k examp. & $50.34 \pm 0.37$ & $46.41 \pm 7.98$ & $25.33 \pm 3.08$ & $50.32 \pm 0.94$ \\
\hline & prototype network & $81.89 \pm 1.75$ & $80.01 \pm 2.66$ & $67.90 \pm 1.53$ & $63.71 \pm 21.1$ \\
\hline & STILTs & $84.02 \pm 0.54$ & $81.73 \pm 0.23$ & $65.28 \pm 5.60$ & $64.66 \pm 2.89$ \\
\hline & UFO-ENTAIL & $85.06 \pm 0.34$ & $83.71 \pm 1.17$ & $73.06 \pm 2.76$ & $74.73 \pm 2.61$ \\
\hline \multirow{4}{*}{ 5-shot } & train on k examp. & $50.20 \pm 0.23$ & $49.24 \pm 6.82$ & $24.50 \pm 2.77$ & $50.18 \pm 0.85$ \\
\hline & prototype network & $81.89 \pm 1.08$ & $81.48 \pm 0.98$ & $67.50 \pm 2.34$ & $73.22 \pm 0.78$ \\
\hline & STILTs & $84.15 \pm 0.47$ & $82.26 \pm 0.56$ & $66.10 \pm 6.72$ & $68.25 \pm 3.49$ \\
\hline & UFO-ENTAIL & $84.84 \pm 0.61$ & $84.82 \pm 1.18$ & $73.30 \pm 2.65$ & $74.59 \pm 2.87$ \\
\hline \multirow{4}{*}{ 10-shot } & train on k examp. & $50.53 \pm 0.99$ & $57.09 \pm 4.04$ & $25.28 \pm 2.35$ & $52.55 \pm 0.99$ \\
\hline & prototype network & $82.12 \pm 0.70$ & $81.83 \pm 0.54$ & $68.48 \pm 2.40$ & $73.28 \pm 1.51$ \\
\hline & STILTs & $84.08 \pm 0.48$ & $82.26 \pm 0.61$ & $67.93 \pm 3.31$ & $71.08 \pm 4.09$ \\
\hline & UFO-ENTAIL & $\mathbf{8 5 . 2 8} \pm \mathbf{0 . 2 7}$ & $86.19 \pm 1.10$ & $74.23 \pm 2.48$ & $\mathbf{7 7 . 5 8} \pm \mathbf{2 . 5 0}$ \\
\hline \multirow{2}{*}{ full-shot } & train on target $\mathrm{d}$ & $79.98 \pm 0.72$ & $95.55 \pm 0.14$ & $80.47 \pm 3.00$ & $90.20 \pm 0.45$ \\
\hline & STILTs (SOTA) & $86.26 \pm 0.23$ & $95.05 \pm 0.19$ & $82.60 \pm 0.64$ & $89.26 \pm 0.38$ \\
\hline
\end{tabular}

Table 1: Applying UFO-EnTAIL to two entailment benchmarks (RTE and SciTail) and two other NLP tasks (question answering (QA) and coreference resolution (Coref.)), each providing $k$ examples $(k=\{1,3,5,10\})$. Numbers for "STILTS (SOTA)" are upperbound performance while using full labeled data; bold numbers are our top numbers when the few-shot hyperparamter $k<=10$.

presume, for $T$, that a related and rich-annotation dataset always exists. As we discussed, the final goal of NLP (or even AI) is to develop a single machine to solve diverse problems. To the end, we try few-shot entailment here as an attempt.

For each downstream NLP task, we provide $k$ examples for helping the learning of the textual entailment system. Next, we describe in detail how some representative NLP problems are converted to be textual entailment. Our work provides a new perspective to tackle these NLP issues, especially given only a couple of labeled examples.

Question Answering. We attempt to handle the QA setting in which only a couple of labeled examples are provided. A QA problem can be formulated as a textual entailment problem-the document acts as the premise, and the (question, answer candidate), after converting into a natural sentence, acts as the hypothesis. Then a true (resp. false) hypothesis can be translated into a correct (resp. incorrect) answer. We choose the QA benchmark
MCTest-500 (Richardson et al., 2013) which releases an entailment-formatted corpus. MCTest500 is a set of 500 items (split into 300 train, $50 \mathrm{dev}$ and 150 test). Each item consists of a document, four questions followed by one correct answer, and three incorrect answers.

Deep learning has not achieved significant success on it because of the limited training data (Trischler et al., 2016) - this is exactly our motivation that applying few-shot textual entailment to handle annotation-scarce NLP problems.

For MCTest benchmark, we treat one question as one example. $K$-shot means we randomly sample $k$ annotated questions (each corresponds to a short article and has four answer candidates). We obtain $k$ entailment pairs for the class "entailment" and $3 k$ pairs for the class "non-entailment". The official evaluation metrics in MCTest include accuracy and $\mathrm{NDCG}_{4}$. Here, we report accuracy.

Coreference Resolution. Coreference resolution aims to cluster the entities and pronouns that 
refer to the same object. This is a challenging task in NLP, and greatly influences the capability of machines in understanding the text.

We test on the coreference resolution benchmark GAP (Webster et al., 2018), a human-labeled corpus from Wikipedia for recognizing ambiguous pronoun-name coreference. An example from the GAP dataset is shown here:

"McFerran's horse farm was named Glen View. After his death in 1885, John E. Green acquired the farm."

For a specific pronoun in the sentence, GAP provides two entity candidates for it to link. To correctly understand the meaning of this sentence, a machine must know which person ("McFerran" or "John E. Green") the pronoun "his" refers to. GAP has such kind of annotated examples of sizes split as $2 \mathrm{k} / 454 / 2 \mathrm{k}$ in train/dev/test. Please note that some examples have both entity candidates as negative (201 in train, 62 in dev and 227 in testing).

In this work, we transform the coreference resolution problem into an entailment problem by replacing the pronoun with each entity candidate. For example, the above example will lead to the following two hypotheses:

"McFerran's horse farm was named Glen View. After McFerran's death in 1885, John E. Green acquired the farm." ["entailment"]

"McFerran's horse farm was named Glen View. After John E. Green's death in 1885, John E. Green acquired the farm." ["non-entailment"]

It is worth mentioning that we append a "s" to the person entity string if the pronoun is one of \{"his", "His", "her", "Her"\}. Otherwise, using the entity string to replace the pronoun directly. Each replacement will yield a hypothesis - the problem ends up being predicting whether this hypothesis is correct or not, given the original sentence.

We randomly choose $k$ examples from train to learn the entailment system; each example will produce two labeled entailment pairs. The GAP benchmark evaluates the F1 score by gender (masculine and feminine) and the overall F1 by combining the two gender-aware F1 scores. We use the official evaluation script and report the overall F1.

\subsection{Results and Analyses}

Table 1 lists the numbers in $k$-shot settings ( $k=$ $\{1,3,5,10\})$ and the full-shot competitor which uses the full labeled data of $T$. To start, the " 0 shot" setting, compared with the "majority or ran- dom" baseline, indicates that using MNLI as training set and test on various target $T$ has already shown some transferability; but this is far behind the SOTA. We are further interested in three main comparisons:

- Comparing UFO-EnTAIL with the typical metric-based meta learning approach: prototypical networks. Interestingly, prototypical network is worse than STILTS on the two entailment benchmarks while mostly outperforming STILTS slightly on QA and coreference tasks. Our system UFOENTAIL consistently surpasses it with big margins. Prototypical network is essentially a nearest neighbor algorithm (Yin, 2020) pretrained on $S$ only. A testing example in $T$ searches for its prediction by comparing with the $T$-specific class representations constructed by the $k$ examples. A pretrained nearest neighbor algorithm does not necessarily work well if $S$ and $T$ are too distinct.

- Comparing UFO-EnTAIL with the SOTA technique STILTs in $k$-shot settings. Our algorithm outperforms the STILTs across all the tasks. Note that STILTs trains on $S$ and the $k$ examples of $T$ sequentially. What STILTS does is to adapt the pretrained space to the target space, guided by $k$ examples. In contrast, UFO-ENTAIL unifies the RoBERTa encoder and the nearest neighbor algorithm by building cross-task class prototypical representations, then tries to train an unified space on $S$ and $T$.

- Comparing UFO-ENTAIL in $k$-shot settings with the full-shot settings. "Full-shot" has two systems: one pretrains on $S$ then fine-tunes on $T$, the other fine-tune on $T$ directly. Generally, we notice that pretraining on $S$ can finally promote the performance (e.g., in RTE and QA) or get similar numbers (e.g., in SciTail and Coreference tasks). Our system by 10-shot even beats the "full-shot, train on target data" with 5.3\% in RTE and is very close to the SOTA number by "full-shot STILTS" (85.28 vs. 86.26). In other three tasks (SciTail, QA, Coref.), although UFO-ENTAIL by 10 -shot hasn't shown better performance than any full-shot settings, its big improvements over other 10-shot baselines across all the tasks ( $\sim 4 \%$ in SciTail, $\sim 6 \%$ in QA and $>4 \%$ in coreference) demonstrate its superiority of handling open NLP problems in fewshot scenarios.

Please keep in mind that the UFO-ENTAIL system for all the reported NLP tasks originated from the same entailment classifier pretrained on MNLI. 


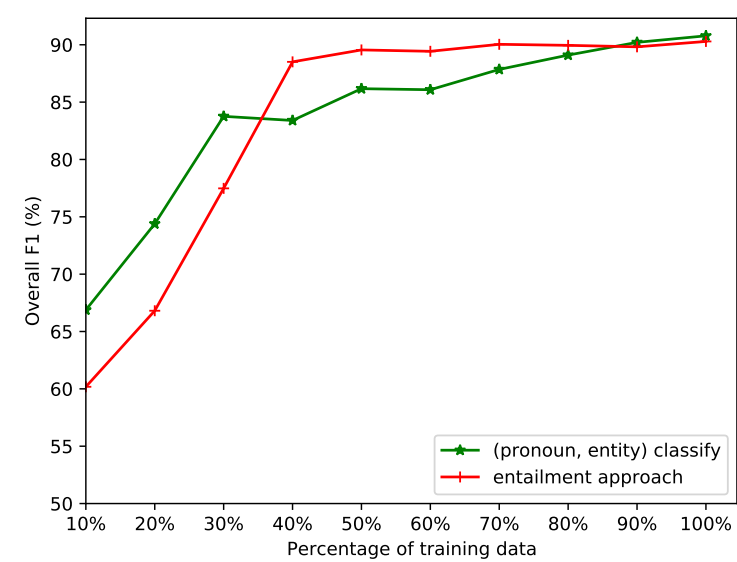

Figure 4: Comparing "entailment" approach with nonentailment approach (i.e., classify (pronoun, entity) pairs in RoBERTa) in coreference's benchmark GAP when using different percentages of training data.

Our experiments indicate: to deal with any open NLP tasks, instead of building large-scale datasets for them separately and let models to fit each of them, it is promising to employ a single entailment system which can generalize well with only a few annotated examples per task.

\subsection{Reformulating NLP problems as textual entailment: better or worse?}

In Table 1, we reported performance of dealing with open entailment and NLP tasks by entailment approach always. We may have another question: for any NLP task, is that better to reformulate it as textual entailment? In this subsection, we compare textual entailment with other popular systems in modeling the coreference task which usually is not modeled in an entailment framework.

To be specific, we feed each instance in the GAP dataset into RoBERTa which will generate a representation for each token in the instance. To obtain representations for the pronoun and an entity candidate, we sum up the representations of all tokens belonging to the pronoun or the entity string. RoBERTa is able to provide the pronoun/entity representations with context in the sentence. Finally, we do binary classification for each (pronoun, entity) pair. We compare this system with the entailment approach (i.e., "train on target data") when using different sizes of training set: [10\%, 20\%, $\cdots, 100 \%]$. To keep a fair comparison, both systems do not pretrain on any other tasks. The result for each percentage is the average of three runs with different seeds.

Figure 4 demonstrates interesting findings: (i)
When using all the GAP training data, both entailment and the (pronoun, entity) classification system reach pretty similar results; (ii) When the training size is below $30 \%$, the non-entailment approach shows better performance. However, the entailment system converges much earlier than the competing system - starting with $40 \%$ training data, it can get performance almost as good as using $100 \%$ data.

This coreference example shows that transforming an NLP task as textual entailment may obtain surprising advantages. There are more NLP tasks that can fit the entailment framework easily, such as text classification (Yin et al., 2019), relation extraction, summarization, etc. However, we also need to admit that reformulating into entailment may also need to fight against new challenges. Taking text classification as an example, how to convert classification labels into hypotheses influences the results a lot. In addition, the hypothesis generation from some NLP tasks may require human efforts to guarantee the quality.

\section{Summary}

In this work, we studied how to build a textual entailment system that can work in open domains given only a couple of examples, and studied the common patterns in a variety of NLP tasks in which textual entailment can be used as a unified solver. Our goal is to push forward the research and practical use of textual entailment in a broader vision of natural language processing. To that end, we proposed utilizing MNLI, the largest entailment dataset, and a few examples from the new domain or new task to build an entailment system via crosstask nearest neighbor. The final entailment system UFO-ENTAIL generalizes well to open domain entailment benchmarks and downstream NLP tasks including question answering and coreference resolution.

Our work demonstrates an example that exploring the uniform pattern behind various NLP problems, enabling us to understand the common reasoning process and create potential for machines to learn across tasks and make easy use of indirect supervision.

\section{Acknowledgments}

The authors would like to thank the anonymous reviewers for insightful comments and suggestions. 


\section{References}

Trapit Bansal, Rishikesh Jha, and Andrew McCallum. 2019. Learning to few-shot learn across diverse natural language classification tasks. CoRR, 1911.03863.

Samuel R. Bowman, Gabor Angeli, Christopher Potts, and Christopher D. Manning. 2015. A large annotated corpus for learning natural language inference. In EMNLP, pages 632-642.

Christopher Clark, Kenton Lee, Ming-Wei Chang, Tom Kwiatkowski, Michael Collins, and Kristina Toutanova. 2019. Boolq: Exploring the surprising difficulty of natural yes/no questions. In Proceedings of NAACL-HLT, pages 2924-2936.

Ido Dagan, Oren Glickman, and Bernardo Magnini. 2005. The PASCAL recognising textual entailment challenge. In Machine Learning Challenges, Evaluating Predictive Uncertainty, Visual Object Classification and Recognizing Textual Entailment, First PASCAL Machine Learning Challenges Workshop, pages 177-190.

Hal Daumé III. 2007. Frustratingly easy domain adaptation. In $A C L$, pages 256-263.

Jacob Devlin, Ming-Wei Chang, Kenton Lee, and Kristina Toutanova. 2019. BERT: pre-training of deep bidirectional transformers for language understanding. In NAACL-HLT, pages 4171-4186.

Suchin Gururangan, Swabha Swayamdipta, Omer Levy, Roy Schwartz, Samuel R. Bowman, and Noah A. Smith. 2018. Annotation artifacts in natural language inference data. In NAACL, pages 107-112.

Xu Han, Hao Zhu, Pengfei Yu, Ziyun Wang, Yuan Yao, Zhiyuan Liu, and Maosong Sun. 2018. FewRel: A large-scale supervised few-shot relation classification dataset with state-of-the-art evaluation. In EMNLP, pages 4803-4809.

Bingyi Kang and Jiashi Feng. 2018. Transferable meta learning across domains. In UAI, pages 177-187.

Nitish Shirish Keskar, Bryan McCann, Caiming Xiong, and Richard Socher. 2019. Unifying question answering and text classification via span extraction. CoRR, abs/1904.09286.

Tushar Khot, Ashish Sabharwal, and Peter Clark. 2018. SciTaiL: A textual entailment dataset from science question answering. In $A A A I$, pages 5189-5197.

Gregory Koch, Richard Zemel, and Ruslan Salakhutdinov. 2015. Siamese neural networks for one-shot image recognition. In ICML deep learning workshop, volume 2 .

Yinhan Liu, Myle Ott, Naman Goyal, Jingfei Du, Mandar Joshi, Danqi Chen, Omer Levy, Mike Lewis, Luke Zettlemoyer, and Veselin Stoyanov. 2019. RoBERTa: A robustly optimized BERT pretraining approach. CoRR, abs/1907.11692.
Bryan McCann, Nitish Shirish Keskar, Caiming Xiong, and Richard Socher. 2018. The natural language decathlon: Multitask learning as question answering. CoRR, abs/1806.08730.

Timothy A. Miller. 2019. Simplified neural unsupervised domain adaptation. In NAACL-HLT, pages 414-419.

Jason Phang, Thibault Févry, and Samuel R. Bowman. 2018. Sentence encoders on stilts: Supplementary training on intermediate labeled-data tasks. CoRR, abs/1811.01088.

Adam Poliak, Aparajita Haldar, Rachel Rudinger, J. Edward $\mathrm{Hu}$, Ellie Pavlick, Aaron Steven White, and Benjamin Van Durme. 2018. Collecting diverse natural language inference problems for sentence representation evaluation. In EMNLP, pages 67-81.

Colin Raffel, Noam Shazeer, Adam Roberts, Katherine Lee, Sharan Narang, Michael Matena, Yanqi Zhou, Wei Li, and Peter J. Liu. 2019. Exploring the limits of transfer learning with a unified text-to-text transformer. CoRR, abs/1910.10683.

Mengye Ren, Eleni Triantafillou, Sachin Ravi, Jake Snell, Kevin Swersky, Joshua B. Tenenbaum, Hugo Larochelle, and Richard S. Zemel. 2018. Metalearning for semi-supervised few-shot classification. In $I C L R$.

Matthew Richardson, Christopher J. C. Burges, and Erin Renshaw. 2013. Mctest: A challenge dataset for the open-domain machine comprehension of text. In EMNLP, pages 193-203.

Tim Rocktäschel, Edward Grefenstette, Karl Moritz Hermann, Tomás Kociský, and Phil Blunsom. 2016. Reasoning about entailment with neural attention. In $I C L R$.

Cícero Nogueira dos Santos, Ming Tan, Bing Xiang, and Bowen Zhou. 2016. Attentive pooling networks. CoRR, abs/1602.03609.

Maarten Sap, Hannah Rashkin, Derek Chen, Ronan Le Bras, and Yejin Choi. 2019. Social iqa: Commonsense reasoning about social interactions. In Proceedings of EMNLP-IJCNLP, pages 4462-4472.

Jake Snell, Kevin Swersky, and Richard S. Zemel. 2017. Prototypical networks for few-shot learning. In NeurIPS, pages 4077-4087.

Flood Sung, Yongxin Yang, Li Zhang, Tao Xiang, Philip H. S. Torr, and Timothy M. Hospedales. 2018. Learning to compare: Relation network for few-shot learning. In $C V P R$, pages 1199-1208.

Adam Trischler, Zheng Ye, Xingdi Yuan, Jing He, and Philip Bachman. 2016. A parallel-hierarchical model for machine comprehension on sparse data. In $A C L$. 
Oriol Vinyals, Charles Blundell, Tim Lillicrap, Koray Kavukcuoglu, and Daan Wierstra. 2016. Matching networks for one shot learning. In NeurIPS, pages 3630-3638.

Alex Wang, Amanpreet Singh, Julian Michael, Felix Hill, Omer Levy, and Samuel R. Bowman. 2019. GLUE: A multi-task benchmark and analysis platform for natural language understanding. In ICLR.

Shuohang Wang and Jing Jiang. 2016. Learning natural language inference with LSTM. In NAACL, pages 1442-1451.

Zhiguo Wang, Wael Hamza, and Radu Florian. 2017. Bilateral multi-perspective matching for natural language sentences. In IJCAI, pages 4144-4150.

Kellie Webster, Marta Recasens, Vera Axelrod, and Jason Baldridge. 2018. Mind the GAP: A balanced corpus of gendered ambiguous pronouns. TACL, 6:605-617.

Adina Williams, Nikita Nangia, and Samuel R. Bowman. 2018. A broad-coverage challenge corpus for sentence understanding through inference. In NAACL-HLT, pages 1112-1122.

Wenpeng Yin. 2020. Meta-learning for few-shot natural language processing: A survey. CoRR, abs/2007.09604.

Wenpeng Yin, Jamaal Hay, and Dan Roth. 2019 Benchmarking zero-shot text classification: Datasets, evaluation and entailment approach. In Proceedings of the 2019 Conference on Empirical Methods in Natural Language Processing and the 9th International Joint Conference on Natural Language Processing (EMNLP-IJCNLP), pages 3905-3914.

Wenpeng Yin and Hinrich Schütze. 2018. Attentive convolution: Equipping cnns with rnn-style attention mechanisms. TACL, 6:687-702.

Mo Yu, Xiaoxiao Guo, Jinfeng Yi, Shiyu Chang, Saloni Potdar, Yu Cheng, Gerald Tesauro, Haoyu Wang, and Bowen Zhou. 2018. Diverse few-shot text classification with multiple metrics. In NAACL-HLT, pages 1206-1215. 